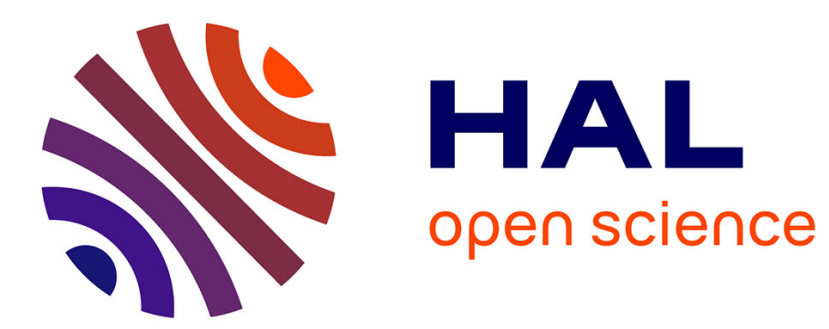

\title{
Solitons in Cyclic and Symmetric Structures
}

Filipe Fontanela, Aurélien Grolet, Loïc Salles, Norbert Hoffmann

\section{To cite this version:}

Filipe Fontanela, Aurélien Grolet, Loïc Salles, Norbert Hoffmann. Solitons in Cyclic and Symmetric Structures. 6th IMAC, A Conference and Exposition on Structural Dynamics 2018, 2018, Orlando, United States. pp.175-178, 10.1007/978-3-319-74280-9_18 . hal-02424294

\section{HAL Id: hal-02424294 \\ https://hal.science/hal-02424294}

Submitted on 27 Dec 2019

HAL is a multi-disciplinary open access archive for the deposit and dissemination of scientific research documents, whether they are published or not. The documents may come from teaching and research institutions in France or abroad, or from public or private research centers.
L'archive ouverte pluridisciplinaire HAL, est destinée au dépôt et à la diffusion de documents scientifiques de niveau recherche, publiés ou non, émanant des établissements d'enseignement et de recherche français ou étrangers, des laboratoires publics ou privés. 


\title{
Solitons in cyclic and symmetric structures
}

\author{
Filipe Fontanela ${ }^{* 1}$, Aurelien Grolet ${ }^{2}$, Loic Salles ${ }^{1}$ and Norbert Hoffmann ${ }^{1,3}$ \\ ${ }^{1}$ Imperial College London, SW7 2AZ, London, UK \\ ${ }^{2}$ Arts et Métiers ParisTech, 8 Boulevard Louis XIV, 59000, Lille, France \\ ${ }^{3}$ Hamburg University of Technology, 21073, Hamburg, Germany
}

\begin{abstract}
This research focuses on localised states arising from modulationally unstable plane waves in non-conservative cyclic and symmetric structures. The main application is on vibrations of bladed-disks of aircraft engines experiencing non-linear effects, such as large displacements, friction dissipation, and/or complex fluid-structure interactions. The investigation is based on a minimal model composed of a chain of linearly damped Duffing oscillators under external travelling wave excitation. The computed results are based on two strategies: (1) a Non-Linear Schrödinger Equation (NLSE) approximation; and (2) the periodic and quasi-periodic Harmonic Balance Methods (HBM). In both cases, the results show that unstable plane waves may self-modulate, leading to stable and unstable single and multiple solitons configurations.
\end{abstract}

Keywords: localised vibrations, solitons, cyclic structures, non-linear Schrödinger equation, harmonic balance methods

\section{Introduction}

Localisation of vibrations is a very important topic in rotating machines, such as bladed-disks of aircraft engines, due to high cycle fatigue. In the linear regime, localised vibrations arise in ideally periodic structures due to inherent inhomogeneities resulting e.g. from the manufacturing processes or wear. However, in real applications, when structures e.g. experience large deformations induced by strong excitations, their behaviours deviate from the linear regime due to non-linearities. It is well-known that, in the non-linear regime, energy localisation may arise even in perfect period structures due to bifurcations.

This research focuses on the non-linear dynamics of cyclic and symmetric structures excited by travelling waves. This excitation is very common in turbomachinery applications since it can be generated due to unbalances or aerodynamic excitations. The findings are based on two different strategies: (1) a non-conservative NLSE approximation; and (2) a fully numeric periodic and quasi-period HBM approach. The results show that stable solitons may emerge from unstable homogeneous solutions, leading to localised vibrations which move along the structure preserving their shapes.

\section{Physical system}

The physical system under investigation consists of $N_{s}$ unitary masses, cyclically connected to each other by linear springs $\omega_{c}^{2}$, and attached to the ground by linear springs $\omega_{0}^{2}$, viscous dampers $\gamma^{2}$, and cubic springs $\xi$. The displacement for the $n$th degree of freedom $u_{n}$ is written as

$$
\ddot{u}_{n}+\gamma^{2} \dot{u}_{n}+\omega_{0}^{2} u_{n}-\omega_{c}^{2}\left(u_{n-1}+u_{n+1}-2 u_{n}\right)+\xi u_{n}^{3}=f_{n},
$$

where $f_{n}$ represents the external excitation. For the following analysis, it is assumed that $f_{n}$ has a travelling wave form such as

$$
f_{n}(t)=F_{0} \exp \left\{\mathrm{i}\left[k(n-1) a-\omega_{f} t\right]\right\}+\text { c.c. }
$$

where $F_{0}$ is the force amplitude, $k$ is the corresponding wave number, $a=2 \pi / N_{s}$ is the lattice parameter, $\omega_{f}$ is the external frequency, $\mathrm{i}$ is the imaginary unit, and c.c. states the complex-conjugate of the first expression. It should be noted that, due to the cyclic symmetry $\left(f_{1}=f_{N_{s}+1}\right), k$ can only assume integer numbers.

\section{Solution approach}

In this section, the two proposed solution approaches are presented. Firstly, the requirements to derive a non-conservative NLSE is introduced, and its limitations are discussed. Finally, a periodic and quasi-periodic HBM is proposed to compute homogeneous solutions and localised states emerging from unstable plane waves, respectively.

\footnotetext{
*Corresponding author: ff515@ic.ac.uk
} 


\subsection{Non-conservative NLSE}

In the weakly non-linear regime the displacement $u_{n}$ is assumed to have the form

$$
u_{n}(t)=\varepsilon \Psi(X, T) \exp \left\{\mathrm{i}\left[k(n-1) a-\omega_{k} t\right]\right\}+\text { c.c. },
$$

where $\Psi(X, T)$ is an envelope function which modulates the travelling wave response, $\omega_{k}=\sqrt{\omega_{0}^{2}+4 \omega_{c}^{2} \sin ^{2}\left(\frac{k a}{2}\right)}$ is the linear natural frequency, and $\varepsilon$ is a small parameter. In Eq. (3), $X$ and $T$ are continuous variables such as $X=\varepsilon x=\varepsilon(n-1) a$ and $T=\varepsilon t$. After plugging Eq. (3) into Eq. (1), it is possible to obtain

$$
\mathrm{i} \frac{\partial \Psi}{\partial \tau}+P \frac{\partial^{2} \Psi}{\partial \eta^{2}}+Q|\Psi|^{2} \Psi=-\mathrm{i} \Gamma \Psi-h \exp \left\{\mathrm{i} \delta_{\omega} \tau\right\}
$$

where $\tau=\varepsilon T$, while $\eta=X-c_{g} t$ is a frame moving with the group velocity $c_{g}=\frac{\mathrm{d} \omega_{k}}{\mathrm{~d} k}$. In Eq (4), the parameter $P=\frac{1}{2} \frac{\mathrm{d}^{2} \omega_{k}}{\mathrm{~d} k^{2}}$ accounts for the dispersion, $Q=-\frac{3 \xi}{2 \omega_{k}}$ is the non-linear term, $\Gamma=\frac{\gamma^{2}}{2}$ models the linear damping effect, $h=\frac{F_{0}}{2 \omega_{k}}$ is the external force, and $\delta_{\omega}=\omega_{k}-\omega_{f}$ is a detuning parameter. The approach is based on a multiscale approximation and the continuous limit [1, 2, 3]. In practical terms, Eq. (4) is valid when: (1) the solution $\Psi$ varies slowly in space and time compared to $a$ and $2 \pi / \omega_{f} ;(2)$ the detuning parameter $\delta_{\omega}$ is small and the system is close to the resonance; and (3) the system weakly forced and weakly damped. Finally, the carrier wave is assumed to vibrate with $\omega_{f}$ such as $\Psi(\eta, \tau)=\psi(\eta, \tau) \exp \left\{\mathrm{i} \delta_{\omega} \tau\right\}$, leading Eq. (4) to an autonomous system.

\subsection{Quasi-periodic HBM}

In the quasi-periodic HBM approach, the solution $u_{n}$ is expanded in a truncated Fourier series such as

$$
u_{n}(t)=\sum_{-k_{2}}^{k_{2}} \sum_{k_{1}}^{k_{2}} A_{k_{2} k_{1}} \cos \left(k_{2} \omega_{2} t+k_{1} \omega_{1} t\right)+B_{k_{2} k_{1}} \sin \left(k_{2} \omega_{2} t+k_{1} \omega_{1} t\right)
$$

where $k_{1}$ and $k_{2}$ are the numbers of retained harmonics, $A_{k_{2} k_{1}}$ and $B_{k_{2} k_{1}}$ are the corresponding amplitudes, while $\omega_{1}$ and $\omega_{2}$ are the two incommensurable frequencies. It is important to notice that if $k_{1}=0$ or $k_{2}=0 \mathrm{Eq}$. (5) recovers the standard periodic HBM. The values of $A_{k_{2} k_{1}}$ and $B_{k_{2} k_{1}}$ are obtained by plugging Eq. (5) into Eq. 11 followed by a Galerkin projection, in order to represent the response in Fourier space such as

$$
[L]\{Z\}+\left\{f_{n l}\right\}-\{g\}=\{0\} .
$$

In Eq. [6], $\{Z\}$ is the vector containing the Fourier coefficients $A_{k_{1} k_{2}}$ and $B_{k_{1} k_{2}},[L]$ is a matrix representing the linear dynamics, while the vectors $\left\{f_{n l}\right\}$ and $\{g\}$ are the projections of the non-linear and the external forces into the Fourier space, respectively (see [4] for a more detailed discussion). The projection from time domain to Fourier space is calculated using a two-dimensional FFT algorithm, where the two frequencies $\omega_{1}$ and $\omega_{2}$ are sampled using a hyper-time approach [4]. Finally, one should note that this paper is interested in quasi-periodic oscillations arising from Neimark-Sacker bifurcations (see Sub. 4). Therefore, the frequency $\omega_{2}$ is an unknown in Eq. (6), and an additional equation representing a phase condition is added to compute the value of $\omega_{2}$ (see [5] for a more detailed discussion).

\section{Numerical Results}

The system described by Eq. (1) is solved assuming $N_{s}=36, \gamma^{2}=0.01 \mathrm{~kg} \cdot \mathrm{s}^{-1}, \omega_{0}^{2}=1.0 \mathrm{~kg} \cdot \mathrm{s}^{-2}, \omega_{c}^{2}=1.0 \mathrm{~kg} \cdot \mathrm{s}^{-2}, \xi=0.10$ $\mathrm{kg} \cdot \mathrm{m}^{-2} \cdot \mathrm{s}^{-2}$. The external force $f_{n}$ is assumed to vibrate with amplitude $F_{0} \sim 0.0225 \mathrm{~N}$ and wave number $k=16 \mathrm{~m}^{-1}$.

In order to solve the previous example with the NLSE approach, standing solutions $\left(\frac{\partial \psi}{\partial \tau}=0\right)$ resulting from the autonomous system discussed in Sub. 3.1 is solved with the continuation code AUTO [6]. Figure 1] shows the maximum displacement of $u$ as a function of $\omega_{f}$. In Panel (a) of Fig. 1 solid lines indicate stable solutions, while dashed lines identify unstable ones (see [7] for the stability analysis of $\psi$ ). Panel (b) of Fig. 1] shows the envelope function $\psi$ for four different points identified in Panel (a): P1, P2, P3 show solutions for the branch detaching around $\omega_{f} \sim 2.2125 \mathrm{rad} / \mathrm{s}$, corresponding to solitons with one hump; and P4 identifies a solution with two humps corresponding to the branch detaching at $\omega_{f} \sim 2.2160 \mathrm{rad} / \mathrm{s}$.

The HBM approach is also applied to the same example using an arc-length continuation [4]. Solutions are shown in Panel (a) of Fig. 1 with dotted blue lines. For the plane wave branch Eq. (5) is solved assuming $k_{2}=0$ and $k_{1}=3$, while the solitons branches are calculated assuming $k_{2}=5$ and $k_{1}=1$. Initial conditions from S1 are used to solve Eq. (1) with a time-marching algorithm. Figure 2 shows the results from the quasi-periodic HBM approach compared to the numerical integration of Eq. (1). The results show great agreement. 
(a)

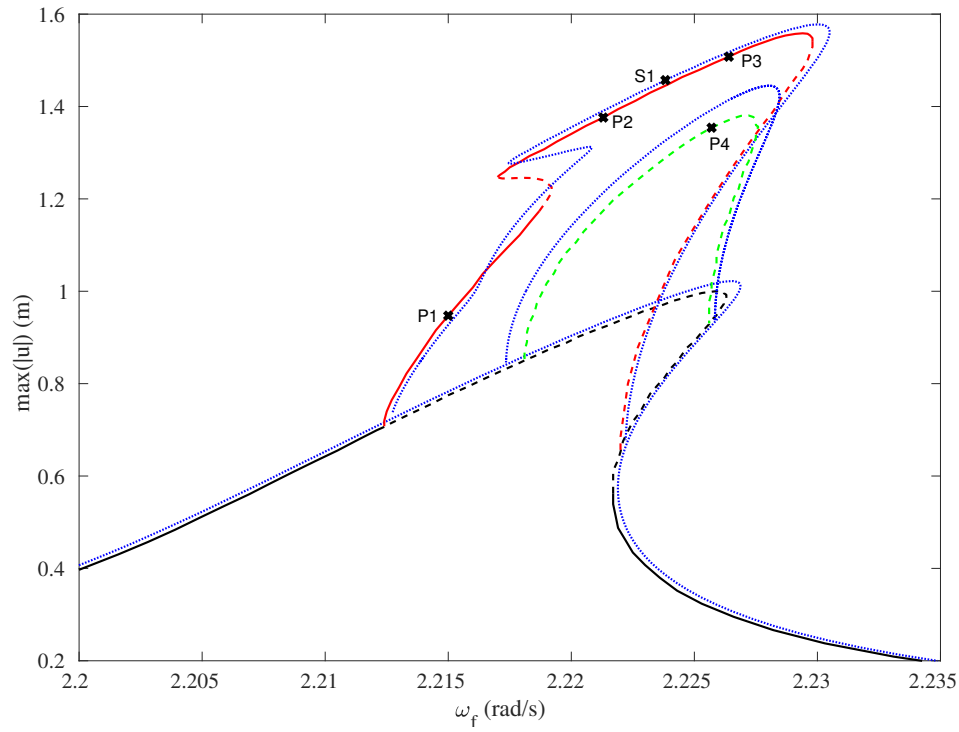

(b)

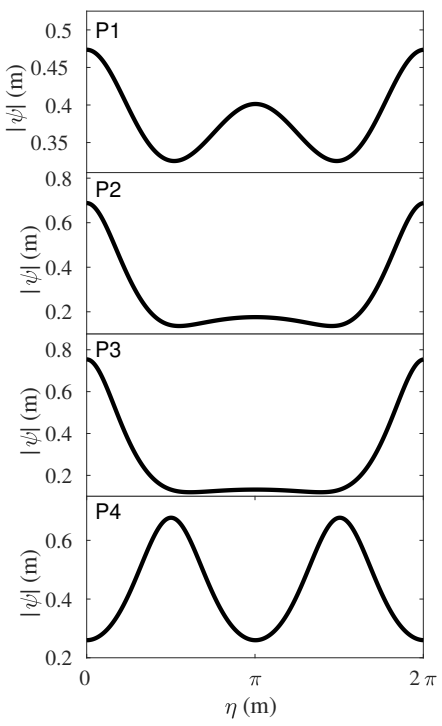

Figure 1: Panel (a) shows the bifurcation diagram computed with the NLSE and the HBM approaches. For the NLSE, solid lines show stable solutions while dashed lines identify unstable ones. Blue dotted lines show results obtained from the HBM approach. Panel (b) shows the envelope functions calculated with the NLSE.

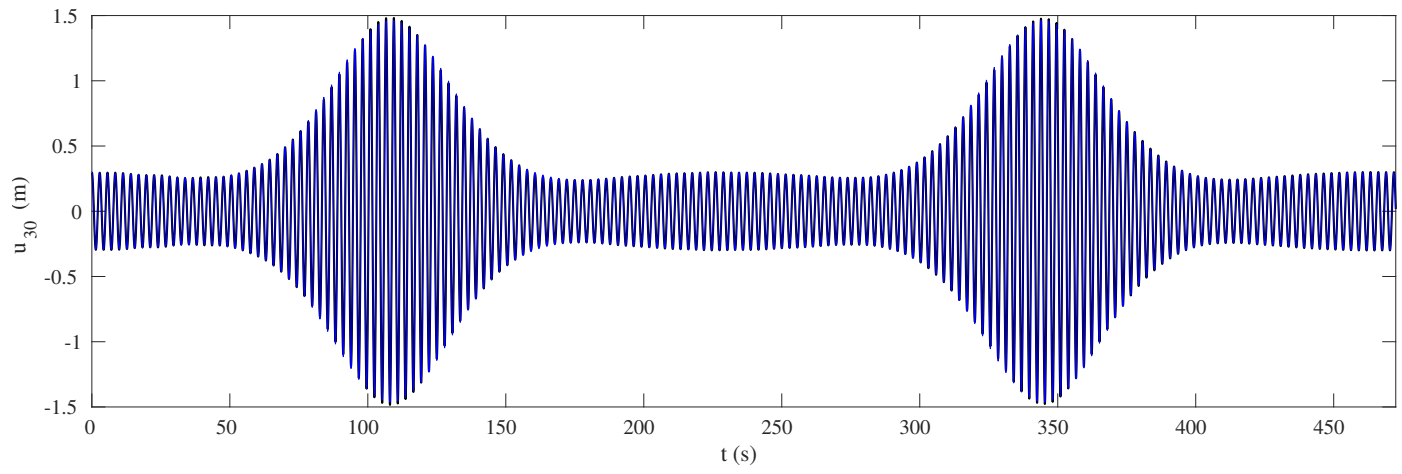

Figure 2: Time evolution of $u_{30}$ calculated with the quasi-periodic HBM (blue) and with a time-marching algorithm (black).

\section{References}

[1] M. Remoissenet, Waves Called Solitons: Concepts and Experiments. Advanced Texts in Physics, Springer, 1994.

[2] A. Grolet, N. Hoffmann, F. Thouverez, and C. Schwingshackl, "Travelling and standing envelope solitons in discrete nonlinear cyclic structures," Mechanical Systems and Signal Processing, vol. 81, no. 15, pp. 1-13, 2016.

[3] F. Fontanela, A. Grolet, L. Salles, A. Chabchoub, and N. Hoffmann, "Dark solitons, modulation instability and breathers in a chain of weakly nonlinear oscillators with cyclic symmetry," Journal of Sound and Vibration, 2017.

[4] M. Guskov and F. Thouverez, "Harmonic Balance-based approach for quasi-periodic motions and stability analysis," Journal of Vibration and Acoustics, vol. 134, no. 3, p. 031003, 2012.

[5] L. Guillot, P. Vigué, C. Vergez, and B. Cochelin, "Continuation of quasi-periodic solutions with two-frequency harmonic balance method," Journal of Sound and Vibration, vol. 394, pp. 434 - 450, 2017.

[6] E. J. Doedel, A. R. Champneys, T. F. Fairgrieve, Y. A. Kuznetsov, B. Sandstede, and X. Wang, "Auto97," Continuation and bifurcation software for ordinary differential equations, 1998.

[7] I. Barashenkov and Y. Smirnov, "Existence and stability chart for the ac-driven, damped nonlinear Schrödinger solitons," Physical Review E, vol. 54, no. 5, pp. 5707-5725, 1996. 